\title{
Synchrotron x-ray topography study of defects in epitaxial GaAs on high-quality Ge
}

\author{
A. Lankinen ${ }^{\text {a }}$ L. Knuuttila ${ }^{\text {a }}$ T. Tuomi ${ }^{\text {a }}$ P. Kostamo ${ }^{\text {a }}$ A. Säynätjoki ${ }^{\text {a }}$ J. Riikonen ${ }^{\text {a }}$ \\ H. Lipsanen ${ }^{\text {a }}$ P. J. McNally ${ }^{b}$ X. Lu ${ }^{\text {b }}$ H. Sipilä ${ }^{\text {c }}$ S. Vaijärvi ${ }^{\text {c }}$ D. Lumb ${ }^{d}$ \\ ${ }^{a}$ Optoelectronics Laboratory, Helsinki University of Technology, P.O. Box 3500, FIN-02015 TKK, Finland \\ ${ }^{\mathrm{b}}$ Research Institute for Networks and Communications Engineering (RINCE), Dublin City University, Dublin 9, Ireland \\ ${ }^{\mathrm{c}}$ Oxford Instruments Analytical Oy, P.O. Box 85, FI-02631 Espoo, Finland \\ dScience Payload and Advanced Concepts Office, ESA/ESTEC, 2200 AG, Noordwijk, The Netherlands
}

\begin{abstract}
Crystal defects of GaAs thin films deposited by metalorganic vapour phase epitaxy on high-quality Ge substrates are studied by synchrotron x-ray topography. The GaAs thin films were measured to have $\approx 500$ dislocations $\mathrm{cm}^{-2}$, which is a similar number to what plain Ge substrates show. The dislocation densities measured are also smaller than for instance those of high-quality vapour pressure controlled Czochralski grown GaAs wafers, which typically have dislocation densities of $\approx 1500 \mathrm{~cm}^{-2}$. The GaAs films grown on both sides of two-sided substrates display very good crystal quality throughout the sample.
\end{abstract}

Key words: Crystal defects, X-ray topography, Heterostructures, Germanium, Gallium arsenide, X-ray detectors PACS: 79.60.Jv, 81.05.Ea, 61.72.Ff, 81.10.Bk

\section{Introduction}

Recently, there has been interest in heteroepitaxial Ge/GaAs material structures for x-ray detectors following the success in GaAs based devices [1]. Infrared detectors based on heteroepitaxy have been previously studied e.g. with InAs/InGaAs combination [2]. In high quality semiconductor devices, especially in those that are heterostructure based, a near-perfect crystal lattice is vital. In this work, crystal defects in thin heteroepitaxial GaAs films are studied by means of x-ray diffraction

Email address: Aapo.Lankinen@iki.fi (A. Lankinen). topography using synchrotron radiation [3]. The GaAs films were deposited by metalorganic vapour phase epitaxy on high purity Ge substrates.

There is a small lattice difference between Ge (lattice constant $5.658 \AA$ at $300 \mathrm{~K}[4]$ ) and GaAs (lattice constant $5.653 \AA$ at $300 \mathrm{~K}[4]$ ). However, GaAs layers not exceeding the critical thickness of the material pair can be free of defects inflicted by the misfit between GaAs and Ge on the lattice. In addition to effects caused by the different layer thicknesses, the formation of misfit dislocations and other defects in the GaAs epilayer is strongly affected by the various parameters used in the growth process. The presence of the lattice 


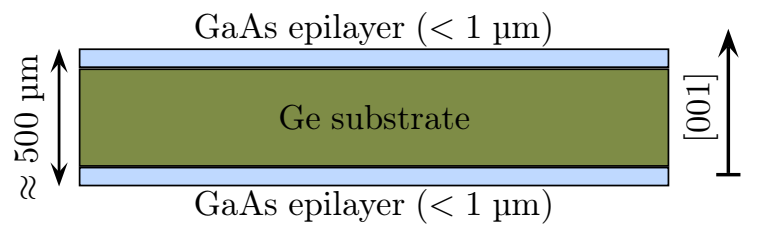

Fig. 1. Schematic drawing of a Ge/GaAs sample. The drawing is not in scale. Some samples had only one of the GaAs epilayers deposited.

defects in the sample is not necessarily revealed if only the sample surface is examined. Therefore a method with a possibility to extract information inside the sample is desirable. X-ray synchrotron topography is a nondestructive diffraction imaging method suited for studying the existence of lattice defects both in the bulk and on the surface of nearly perfect single crystals.

\section{Experimental}

Figure 1 shows a schematic drawing of a $\mathrm{Ge} / \mathrm{GaAs}$ sample. The Ge substrates were (001) $6^{\circ}$ misoriented $500 \mu \mathrm{m}$ thick Czochralski-grown n and $\mathrm{p}$ type wafers, on top of which the GaAs layers were grown at Optoelectronics Laboratory using metal organic vapour phase epitaxy (MOVPE). Some of the samples had an epilayer deposited on both sides of the samples, because the planned detector structure would use the Ge substrate as an active layer between the epilayers. The substrate temperature in epitaxial growth was about $530^{\circ} \mathrm{C}$ to $620^{\circ} \mathrm{C}$ depending on other growth parameters. Tertiarybutylarsine (TBAs) and trimethylgallium (TMGa) were used as precursors. The epitaxial layers examined had thicknesses varying from few tens of nanometers to about $1 \mu \mathrm{m}$.

The x-ray diffraction topographs were made at the HASYLAB-DESY (Hamburger Synchrotronstrahlungslabor am Deutschen ElektronenSynchrotron) in Hamburg at the F1 topography beam line using the radiation of the DORIS bending magnet source having a continuous spectrum of wavelengths. Topographs were recorded on $100 \mathrm{~mm} \times 100 \mathrm{~mm}$ Slavich VPR-M high-resolution films both in the transmission and in the backreflection geometry with a film-to-sample distance a)

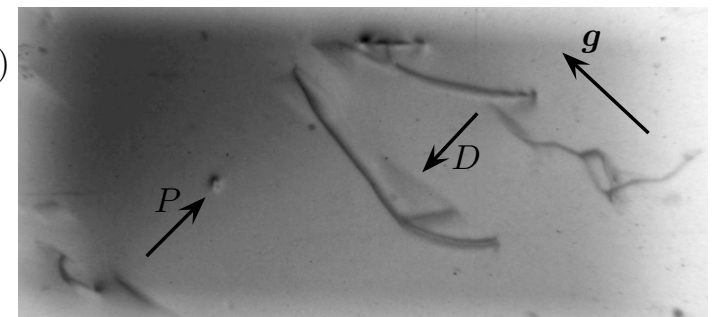

b)

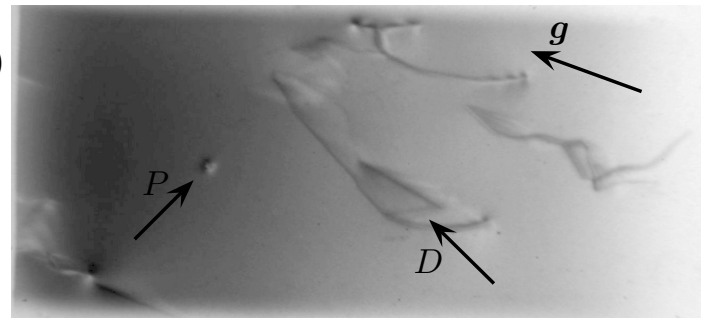

Fig. 2. a) $5 \overline{1} 1$ and b) 511 Large area transmission topographs of a Ge/GaAs sample showing dislocations in Ge substrate and GaAs epilayer. Dislocation density in both Ge and GaAs was measured to be $250-500 \mathrm{~cm}^{-2}$, and no additional dislocations were produced by GaAs growth. Disappearing images of threading dislocations having Burgers vectors of type $\langle 011\rangle$ are marked with arrows $D$ and a precipitate with $P$. Projections of the diffraction vectors $\boldsymbol{g}$ are shown in top right corners of the topographs. Image size is $2 \mathrm{~mm} \times 1 \mathrm{~mm}$ for both topographs.

of $60-80 \mathrm{~mm}$. The (001) surfaces of the samples were set perpendicularly to the incident beam in both back-reflection and transmission topography. The epitaxial layer was on the film side in all exposures.

\section{Results and discussion}

\subsection{Crystal quality and dislocation densities}

Figures 2 a) and b) show $5 \overline{1} 1$ and 511 large area transmission topographs of a $210 \mathrm{~nm}$ thick GaAs film on a Ge substrate. The topographs contain images of mixed type threading or circular arc dislocations having Burgers vectors of type $\langle 011\rangle$, but these dislocations are also observed in bulk Ge wafers with the same dislocation density of about $250-500 \mathrm{~cm}^{-2}$. Thus, the epilayer growth process does not optimally produce any additional dislocations into the structure. 


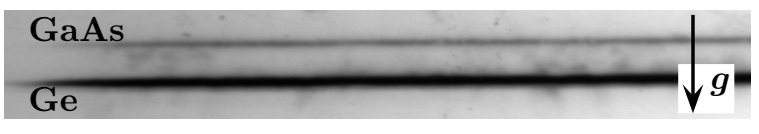

Fig. 3. 117 back-reflection section topograph of a Ge/GaAs sample having $600 \mathrm{~nm}$ thick epilayer. Because the GaAs epilayer is strained, its section image is shifted above the substrate section image. Diffraction vector is marked with $\boldsymbol{g}$. Image width is $2 \mathrm{~mm}$.

The observed dislocation density is less than for instance that of high quality vapour pressure controlled Czochralski (VCz) grown GaAs wafers (typically $\approx 1500 \mathrm{~cm}^{-2}[5]$ ), and also the overall defect density of the $\mathrm{Ge} / \mathrm{GaAs}$ heterostructure is very low. No stacking faults were found in the topographs.

Figure 3 shows a 117 back-reflection section topograph of a $600 \mathrm{~nm}$ thick GaAs film on Ge. The GaAs film image is focused and it is clearly separated from the Ge substrate image, which indicates that no relaxation has occurred in the GaAs layer. The section topograph does not show any additional defect images when compared to the topograph of the $210 \mathrm{~nm}$ thick layer in Fig. 2.

\subsection{Precipitates}

Enlargements of transmission topographs presented in Figs. 4 a) $\overline{5} \overline{1} 1$ b) $\overline{5} 11$ c) 511 and d) 531 show images of an extraordinary precipitate found in the sample having the $210 \mathrm{~nm}$ thick GaAs epilayer. The precipitate images in the enlargements are from the same film as the topographs in Fig 2, where the precipitate image is marked with $P$. The topographs indicate that the strain field of the precipitate is radially symmetric, but the innermost volume around the precipitate core does not produce contrast in the topograph. It is believed that the core of the precipitate is less strained.

Almost similar topographs of precipitates have been discussed in literature, and they were speculated to be caused by diffusion of impurities into the substrate [6]. It is possible that the precipitate seen in the Ge/GaAs sample is similarly formed by e.g. arsenic diffusion into Ge. However, it is also possible that the precipitate is at least partially in the epitaxial GaAs layer. In any case, the Ge wafers without GaAs epilayer do not show precip- a)

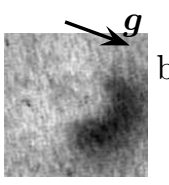

b)
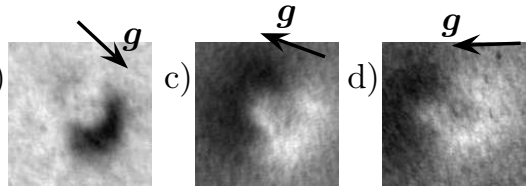

Fig. 4. a) $\overline{5} \overline{1}$ b) $\overline{5} 11$ c) 511 and d) 531 large area topograph enlargements of a precipitate in a $\mathrm{Ge} / \mathrm{GaAs}$ sample with the $210 \mathrm{~nm}$ epilayer. Diffraction vectors are marked with $\boldsymbol{g}$. Image size is $100 \mu \mathrm{m} \times 100 \mu \mathrm{m}$ for all the enlargements.

itates, so it is evident that the precipitates are the result of the epitaxial growth process.

It should be noted that the feature seen in the topographs of Fig. 4 is the image of the strain field produced by the precipitate, which is much larger than the precipitate itself. The sizes of arsenic precipitates in GaAs typically are within a range of few tens of $\mathrm{nm}$ to $\approx 0.6 \mu \mathrm{m}[7]$.

\subsection{Critical thickness}

Figures 5 a) and b) show large area transmission and back-reflection topographs of a $\mathrm{Ge} / \mathrm{GaAs}$ sample having a film thickness of $750 \mathrm{~nm}$. Both topographs display a clear misfit dislocation network, which indicates that the critical thickness is exceeded. The critical thickness for the sample series examined was measured to be between $650 \mathrm{~nm}$ and $750 \mathrm{~nm}$. The transmission topograph in Fig. 5 a) also shows threading dislocations.

Theoretically the critical thickness for a lattice difference of $0.8 \%$ should be about $200 \mathrm{~nm}$ [8], which is slightly less than $290-450 \mathrm{~nm}$ found in a previous experimental work [9]. However, the critical thickness observed in this work (>650 nm) was over three times the expected value for the $\mathrm{Ge} / \mathrm{GaAs}$ structures studied. The difference is believed to be partly caused by the lower growth temperature reducing the thermal stress in the growth process. The observed relatively large critical thickness is expected to improve the possibility to use $\mathrm{Ge} / \mathrm{GaAs}$ structures in x-ray detectors.

\section{Conclusion}

The best of the Ge/GaAs samples examined were found to have an excellent crystal quality, 
a)

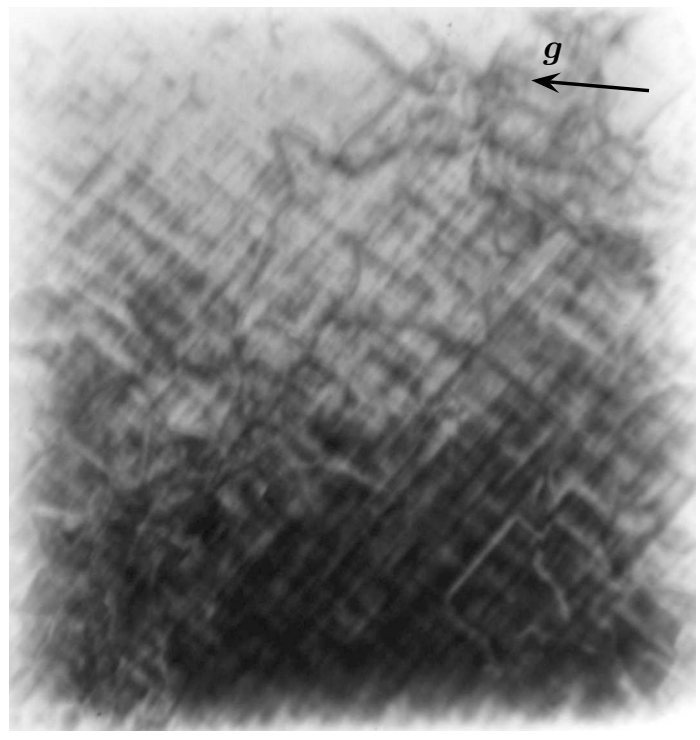

b)

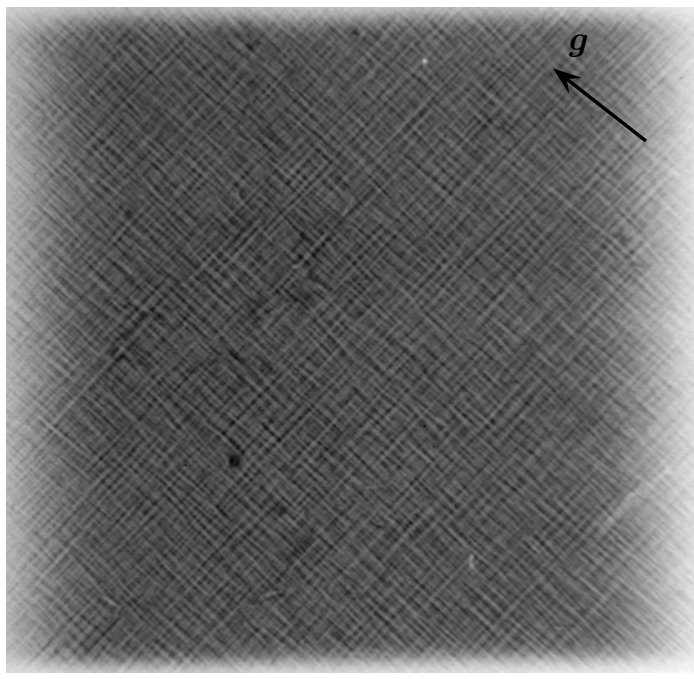

Fig. 5. a) $5 \overline{1} 1$ large area transmission and b) 004 large area back-reflection topographs of a sample having $750 \mathrm{~nm}$ GaAs epilayer on Ge substrate. The sample displays a misfit dislocation network between Ge substrate and GaAs epilayer. Diffraction vectors are marked with $\boldsymbol{g}$. Image size is $1.6 \mathrm{~mm} \times 1.6 \mathrm{~mm}$ for both topographs.

even when the theoretical critical thickness was exceeded. The crystal quality in the thin films studied is believed to be adequate for x-ray detectors based on Ge/GaAs heterostructures. Particularly, the lowest dislocation density was as low as $250-500 \mathrm{~cm}^{-2}$, which is less than is found in typical GaAs wafers. Some precipitates were found in the samples.

\section{Acknowledgements}

This work was supported by the European Space Agency Contract 17356/03/NL/CP and by the IHP-Contract HPRI-CT-2001-00140 of the European Commission.

This work was also supported by the European Community - Research Infrastructure Action under the FP6 "Structuring the European Research Area" Programme (through the Integrated Infrastructure Initiative "Integrating Activity on Synchrotron and Free Electron Laser Science".)

\section{References}

[1] A. Owens, M. Bavdaz, A. Peacock, H. Andersson, S. Nenonen, M. Krumrey and A. Puig, Nuclear Instruments and Methods in Physics Research A, 479, (2002): 531-534.

[2] P. J. McNally, J. Curley, A. Krier, Y. Mao, J. Richardson, T. Tuomi, M. Taskinen, R. Rantamäki, E. Prieur and A. Danilewsky, Semicond. Sci. Technol., 13, (1998): 345-349.

[3] T. Tuomi, K. Naukkarinen and P. Rabe, physica status solidi (a), 25, (1974): 93-106.

[4] O. Madelung (Ed.), Data in Science and Technology, Semiconductors Group IV Elements and III-V Compounds, Springer, Berlin, 1991.

[5] T. Tuomi, L. Knuuttila, J. Riikonen, P. J. McNally, W.-M. Chen, J. Kanatharana, M. Neubert and P. Rudolph, Journal of Crystal Growth, 237-239, (2002): $350-355$.

[6] B. K. Tanner, X-ray Diffraction Topography, Pergamon, 1976, pp. 84-86.

[7] J. L. Weyher, T. Schobera, K. Sonnenberga and P. Franzosib, Materials Science and Engineering B, 55, (1998): 79-85.

[8] T. King-Ning, J. W. Mayer and L. C. Feldman, Electronic Thin Film Science For Electrical Engineers and Materials Scientists, Macmillan Publishing Company, New York, 1992, pp. 170-174.

[9] G. Attolini, C. Bocchi, P. Franzosi, D. Korytar and C. Pelosi, Journal of Physics D: Applied Physics, 28, (1995): A129-A132. 